\title{
Porcine and Human In Vivo Simulations for Doxorubicin-Containing Formulations Used in Locoregional Hepatocellular Carcinoma Treatment
}

\author{
Ilse R. Dubbelboer, ${ }^{1}$ (i) Erik Sjögren, ${ }^{1}$ and Hans Lennernäs ${ }^{1,2}$
}

Received 20 April 2018; accepted 31 July 2018; published online 30 August 2018

\begin{abstract}
It is important to be able to simulate and predict formulation effects on the pharmacokinetics of a drug in order to optimize effectivity in clinical practice and drug development. Two formulations containing doxorubicin are used in the treatment of hepatocellular carcinoma (HCC): a Lipiodol-based emulsion (LIPDOX) and a loadable microbead system (DEBDOX). Although equally effective, the formulations are vastly different, and little is known about the parameters affecting doxorubicin release in vivo. However, mathematical modeling can be used to predict doxorubicin release properties from these formulations and its in vivo pharmacokinetic (PK) profiles. A porcine semiphysiologically based pharmacokinetic (PBPK) model was scaled to a human physiologically based biopharmaceutical (PBBP) model that was altered to include HCC. DOX in vitro and in vivo release data from LIPDOX or DEBDOX were collected from the literature and combined with these in silico models. The simulated pharmacokinetic profiles were then compared with observed porcine and human HCC patient data. DOX pharmacokinetic profiles of LIPDOX-treated HCC patients were best predicted from release data sets acquired by in vitro methods that did not use a diffusion barrier. For the DEBDOX group, the best predictions were from the in vitro release method with a low ion concentration and a reduced loading dose. The in silico modeling combined with historical release data was effective in predicting in vivo plasma exposure. This can give useful insights into the release method properties necessary for correct in vivo predictions of pharmacokinetic profiles of HCC patients dosed with LIPDOX or DEBDOX.
\end{abstract}

KEY WORDS: DC bead; doxorubicin; hepatocellular carcinoma; in vitro-in vivo correlation; Lipiodol; physiologically based pharmacokinetic modeling; physiologically based biopharmaceutical modeling; TACE.

\section{INTRODUCTION}

Drug development is aided by correct predictions of the formulation effects on the in vivo pharmacokinetic (PK) profiles of the active pharmaceutical ingredients. Highly predictive in silico tools for this purpose may reduce the costs and risks of the drug development process $(1,2)$. One frequently used in silico methodology to describe drug disposition is physiologically based pharmacokinetic (PBPK) modeling (2). The PBPK models focus on drug disposition, e.g., distribution and elimination. Another method, physiologically based biopharmaceutical (PBBP) modeling, also involves physiological information.

Electronic supplementary material The online version of this article (https://doi.org/10.1208/s12248-018-0251-4) contains supplementary material, which is available to authorized users.

\footnotetext{
${ }^{1}$ Department of Pharmacy, Uppsala University, Box 580, 75123 , Uppsala, Sweden.

${ }^{2}$ To whom correspondence should be addressed. (e-mail: hans.lennernas@farmaci.uu.se)
}

However, PBBP modeling focuses on the effects of the formulation properties on the PK of a drug, i.e., the characteristics of the formulation, its interaction with the physiological or pathophysiological environment, and the release of the drug from the formulation (3). Several PBPK/PBBP tools have the possibility to simulate oral administration of oral dosage forms, where formulation and drug properties obtained from in vitro methods are used (4). However, for non-oral formulations, there are no standardized, biopharmaceutical in vitro release methods to determine the release of an active pharmaceutical ingredient (5). These non-oral formulations include the parenteral drug delivery systems (DDS) used in the locoregional treatment of intermediate hepatocellular carcinoma (HCC).

Primary liver cancer, of which HCC accounts for $70-85 \%$, is the second-most common cause of death from cancer $(6,7)$. The incidence is increasing all over the world, and in 2012, 745,000 people were diagnosed with primary liver cancer (7). The treatment of HCC depends on its stage (8). Intermediate HCC is treated by infusion of the parenteral DDS into the hepatic artery leading to, or into, the tumor-feeding artery (9). 
This administration procedure is called transarterial chemoembolization (TACE). The injectable DDS usually causes an embolization in the artery, while simultaneously releasing the drug into the surrounding blood and tissue. The rate and extent of drug release varies, depending on the pharmaceutical properties of the DDS (9). Two commonly used DDSs in this context are (i) an emulsion (LIPDOX) composed of Lipiodol (LIP) and a solution containing doxorubicin (DOX), and (ii) a drug-eluting bead (DEB, here DC Bead) loaded with DOX (DEBDOX) $(9,10)$. The composition of the LIPDOX emulsion is clinic dependent, which affects the stability of the emulsion and thus the release rate (11-13). DEBDOX is a more defined product, with an extended release rate (9). These two vastly different DDSs have been evaluated in multiple studies by different in vitro and in vivo release methods (14-18). Factors affecting the in vitro release are well known, but how well do these data correlate with the in vivo release?

This paper is a continuation in the ongoing liver cancer project on the investigation of LIPDOX and DEBDOX from (bio)pharmaceutical perspectives. The project has already resulted in a multitude of articles using in vitro (19), in vivo $(11,17,20,21)$, and in silico (22) techniques, and even in a public discussion with one of the manufacturers $(12,13)$. The work presented here carries on from the development of our porcine semi-PBPK model for DOX and DOXol (22). Two models were fitted to observed pig data: a generic and a binding-specific PBPK model. It was clear only the binding-specific model was able to describe the prolonged elevated tissue concentrations of DOX and DOXol (22). In the PBPK model fittings, disposition parameters for DOX and DOXol were estimated. These disposition parameters were estimated by curve fitting to observed data after intravenous administration of DOX from the non-clinical pig study 1 ( $\mathrm{NC1})$, where the in vivo DOX release from LIPDOX and DEBDOX was investigated (17), and the non-clinical pig study 2 (NC2) where the effect of Lipiodol on DOX disposition was examined $(20,21)$.

The aim of this report was to simulate the DOX plasma concentration-time profiles of $\mathrm{HCC}$ patients treated locoregionally with LIPDOX or DEBDOX and to compare these to observed patient data. Our previously published semi-PBPK model for DOX and its active metabolite doxorubicinol (DOXol) in healthy pigs (22) was further developed into a PBBP model for healthy pigs and human $\mathrm{HCC}$ patients. In vivo $\mathrm{PK}$ profiles in healthy pigs and $\mathrm{HCC}$ patients were simulated using in vitro DOX release data from LIPDOX and DEBDOX formulations to determine the best descriptive in vitro release method for either DDS.

\section{METHODS}

Firstly, physiologically based biopharmaceutical (PBBP) models for healthy pigs and human HCC patients receiving locoregional treatment with LIPDOX or DEBDOX (Fig. 1) were developed from the previously developed porcine semi-PBPK model (22). The purpose of the PBBP model is to describe the pharmacokinetics of DOX, and to evaluate which historical release profile best describes the in vivo release rate. The development of the PBBP models is extensively described in the supplementary information, together with the results of and discussion on the model development (Electronic supplementary material 1). In short, the semi-PBPK model was adapted to better reflect the GI tract binding capacity observed. Thereafter, the liver was divided into two compartments: a treated and untreated compartment, reflecting the sections of liver where the DDS is administered. By adding an administration site for DOX to the treated liver section, the PBPK model was developed into a PBBP model. The pig PBBP model was translated to human, by using human physiology. In the next step, the option to include liver cirrhosis was added. And finally, functionality of the treated liver section was adapted to reflect a HCC tumor. These models were used to simulate the DOX plasma concentration-time profiles of healthy pigs and human HCC patients in the remainder of this report.

\section{Collection and Processing of LIPDOX and DEBDOX Release Data}

A total of 53 historical in vitro and in vivo release data sets of DOX from LIPDOX and DEBDOX were collected (Electronic supplementary material 2); 25 of these were for LIPDOX (LIP1-25), and 28 for DEBDOX (DEB1-28). These data sets were obtained either through personal communication (19)[Ahnfelt, personal communication] or extracted from published reports using PlotDigitizer (Free Software Foundation Inc., MA, USA) (14,16-18,23-28).

DOX release parameters for the LIP and DEB data sets were estimated by curve fitting a modified version of the Weibull equation (Eq. 1) to the obtained historical release profiles. This equation was chosen as it is able to fit to different curve shapes (29).

$A_{\text {rel }}=\operatorname{dose} * F_{\text {rel,max }} *\left(1-e^{-A t^{B}}\right)$

where $A_{\text {rel }}$ is the cumulative amount/fraction/percentage released into the medium at a specific time $(t)$; dose is the amount/fraction/percentage that is loaded into the DDS; $F_{\text {rel,max }}$ is the maximum fraction of the dose that can be released from the formulation; and $A$ and $B$ are constants affecting the shape of the release curve.

\section{Porcine DOX and DOXol PK Profile Simulations}

Porcine DOX and DOXol plasma concentration-time and biliary excretion profiles were simulated using the porcine PBBP model (Electronic supplementary material 1). Physiological and substance-specific parameters for the porcine PBBP model are equal to the previously published semi-PBPK model (22), i.e., no physiological or substance-specific parameters were estimated in this report. Two simulations were performed for each historical release data set, one simulation with disposition parameters derived from the NC1 study, and one simulations with disposition parameters derived from the NC2 study. These disposition parameters were previously published with our semi-PBPK model (22). Release of DOX from LIPDOX and DEBDOX $\left(R_{\mathrm{DDS}}\right.$; $\mu \mathrm{mol} / \mathrm{min}$ ) into the vascular compartment of the treated liver section is described by a derivative of the Weibull function (Eq. 2):

$R_{\mathrm{DDS}}=\operatorname{dose} * F_{\text {rel, } \max } * A * B * t^{B-1} * e^{-A t^{B}}$

The release parameters $\left(A, B\right.$, and $\left.F_{\text {rel,max }}\right)$ were obtained from the curve fittings of the LIP1-25 and DEB1-28 data sets in the previous section. Simulations were performed with the administered doses in the LIPDOX and DEBDOX arms of the NC1 pig study (17). Four pigs received LIPDOX in a 1:3.3 aq:Lipiodol ratio, containing a total of $50 \mathrm{mg}$ DOX. Unexpected 

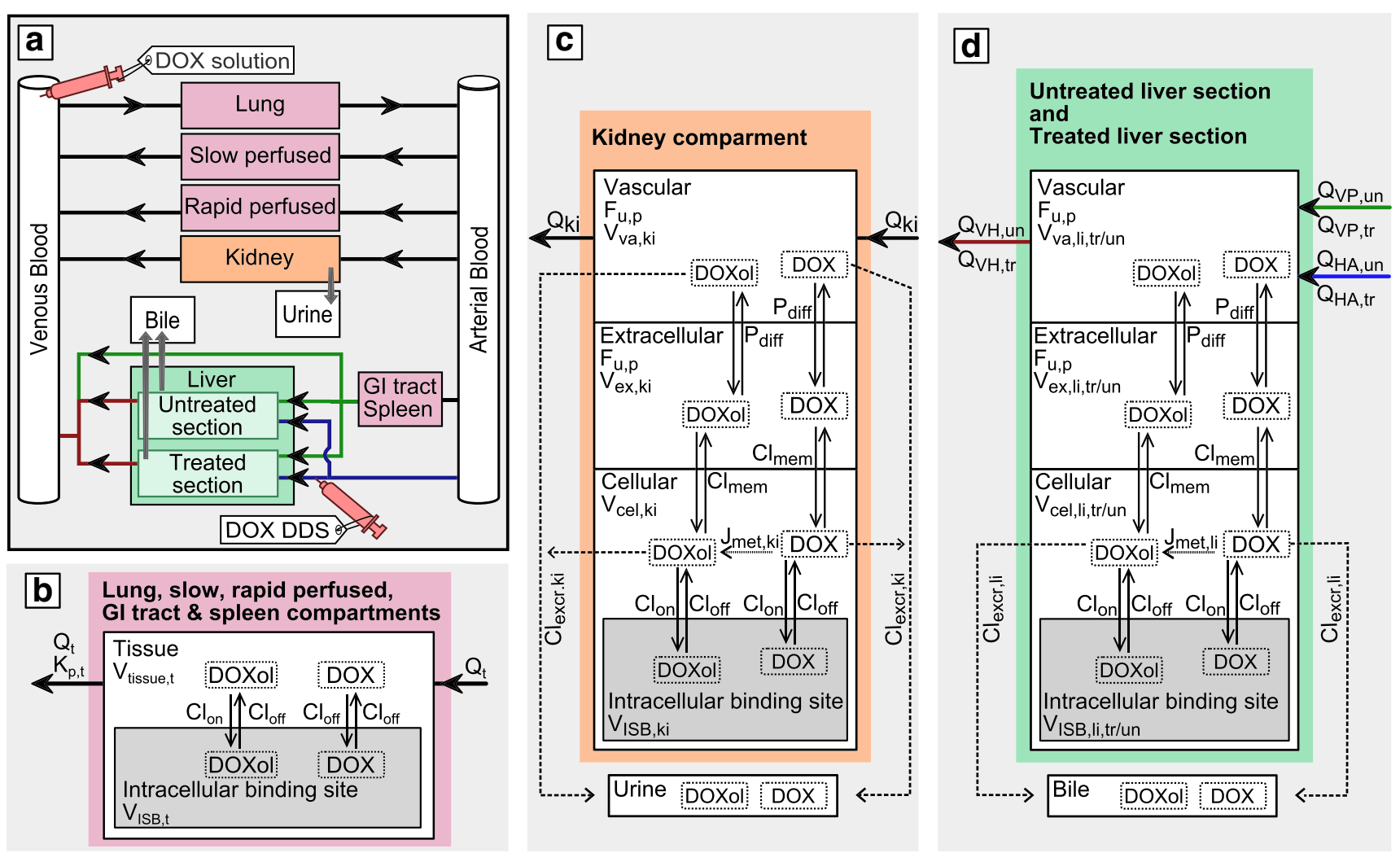

Fig. 1. The structure of the physiologically based biopharmaceutical (PBBP) model for doxorubicin (DOX), and its active metabolite doxorubicinol (DOXol), is depicted in (a). The model consisted of ten compartments (boxes) - six for tissue, two for blood, and two for excretion. The distribution of the drug into the tissue compartments is depicted for non-eliminating, non-metabolizing tissues (pink, b), kidney (orange, c), and liver (green, d). In each of the tissue compartments, intracellular binding is possible to the intracellular binding site (gray box). All tissues have blood flow (Q), and a tissue volume (V). Both kidney (c) and liver (d) comprise three subcompartments, i.e., vascular, extracellular, and cellular, where the flux of the drug between them is described by passive diffusion $\left(P_{\text {diff }}\right)$ and cellular membrane clearance $\left(\mathrm{Cl}_{\text {mem }}\right)$. In both kidney $(\mathbf{c})$ and liver $(\mathbf{d})$, DOX and DOXol are excreted $\left(\mathrm{Cl}_{\text {excr }}\right)$ to urine or bile, and DOX is metabolized to DOXol $\left(J_{\text {met }}\right)$. The liver compartment is split into treated and untreated sections (d), where the size of the sections could be varied. DOX solution (burgundy syringe) was administered to the venous blood compartment, or to the vascular subcompartment of the treated liver section. $\mathrm{Cl}_{\text {excr }}$, excretion clearance to bile or urine; $\mathrm{Cl}_{\text {mem }}$, cell membrane clearance in liver and kidney; $\mathrm{Cl}_{\text {off }}$, dissociation clearance from intracellular binding site; $\mathrm{Cl}_{\mathrm{on}}$, association clearance to intracellular binding site; DOX, doxorubicin; DOXol, doxorubicinol; $\mathrm{F}_{\mathrm{u}, \mathrm{p}}$, fraction unbound plasma; GI, gastrointestinal tract; HA, hepatic artery; ISB, intracellular binding site; $\mathrm{J}_{\text {met }}$, corrected total metabolic capacity; ki(,b/u), bound/unbound concentration of substance in kidney; $\mathrm{li}(, \mathrm{b} / \mathrm{u})$, bound/unbound concentration of substance in liver; $\mathrm{P}_{\mathrm{diff}}$, passive diffusion between vascular and extracellular space in liver and kidney; $\mathrm{Q}$, blood flow; $\mathrm{t}(\mathrm{b} / \mathrm{u})$, bound/unbound concentration of substance in tissue; tr, treated. un, untreated; V, volume; VH, hepatic vein; VP, portal vein. Adapted with permission from (22). Copyright 2017 American Chemical Society.

early stasis was observed in this treatment group, hampering the administration of the total dose. Only one pig had measurable DOXol plasma concentrations, and this one had received $9.9 \mu$ mole $(5.6 \mathrm{mg})$ DOX. Therefore, simulations were performed with this dose and compared to observations from this one animal. In the DEBDOX arm, four pigs received DEBDOX ${ }_{100-300 \mu m}$ loaded with $25 \mathrm{mg} / \mathrm{ml}$. Therefore, the DEBDOX simulations were performed with a dose of $92 \mu$ mole $(50 \mathrm{mg})$.

Stasis of blood flow caused by LIPDOX and DEBDOX was described in the simulations. After administration of $0.1 \mathrm{~mL} / \mathrm{kg}$ LIP to rats, about $50 \%$ of the sinusoids regain normal blood flow after 1 day, and complete blood flow after 3 days (30). Thus, when simulating administration with LIPDOX, the blood flow to the treated area was described by the following equation:

$Q_{\mathrm{tr}, \mathrm{emb}}=Q_{\mathrm{tr}} * F_{\mathrm{vol}, \mathrm{tr}} *\left(1-e^{-k * t}\right)$

where $Q_{\operatorname{tr}(\text { emb) }}$ is the (reduced) blood flow to the treated area; $F_{\mathrm{vol}, \text { tr }}$ is the volume fraction of treated section (0.0517); and $t$ is time in min. and $k$ is a value that describes the increase in blood flow to, or decrease in embolization of, the treated section. It is calculated by the following equation in which the time to blood flow recovery ( 3 days) is divided by 7 , as this gives $>99 \%$ blood flow recovery.

$$
k=\frac{\ln (2)}{\text { time to blood flow recovery } / 7}
$$

DEBDOX causes a complete and irreversible embolization in the treated hepatic artery $\left(\mathrm{HA}_{\mathrm{tr}}\right.$, Fig. 1) after administration. However, the treated section is often supplied by two or more hepatic arteries $(31,32)$, and DEBDOX will most likely embolize the vessel used for the administration of DEBDOX. In line with this, a permanent embolization in the treated hepatic artery of $50 \%$ from the first administration minute was applied during simulations with DEBDOX administration. 
The simulations were compared to observed porcine plasma concentration-time for DOX and DOXol, and biliary excretion profiles from the NC1 study (17). The agreement between the simulations and observed data was evaluated visually, and by absolute average fold error (AAFE, Eq. 7; section Data Analysis and Used Software) and average fold error (AAFE, Eq. 8; section Data Analysis and Used Software). Simulations were assumed acceptable if simulations followed observed profiles and the AAFE was $<2.5$ for at least 5 of the 8 profiles [DOX and DOXol in plasma (vena hepatica (VH), vena porta (VP), vena femoralis (VF)) and bile]. Simulations were adequate if there were at least $4 \mathrm{PK}$ profiles with an $\mathrm{AAFE}<2.5$ for both the NC1 and NC2 disposition parameters.

By comparing PK profiles simulated with $\mathrm{NC1}$ or $\mathrm{NC} 2$ disposition parameters (for each of the $8 \mathrm{PK}$ profiles for each in vitro data set), the best descriptive disposition parameter set was acquired. This disposition data set was then used in the remainder of the work.

\section{Human HCC DOX PK Profile Simulations}

Using the human PBBP model, human in vivo PK profiles were simulated after a single administration of LIPDOX or DEBDOX. LIPDOX and DEBDOX intrahepatic dosing were simulated using release parameters from the LIP and DEB data sets which had given adequately described porcine PK profiles (i.e., at least $4 \mathrm{PK}$ profiles with $\mathrm{AAFE}<2.5$ for both $\mathrm{NC1}$ and NC2 disposition parameters). Release of DOX from the DDS $\left(R_{\mathrm{DDS}}, \mu \mathrm{mol} / \mathrm{min}\right)$ into the vascular space of the treated liver section is described using Eqs. 5 and 6.

$R_{\mathrm{DDS}, t=i}=\frac{\text { dose }}{\text { inftime }} * F_{\text {rel, } \max } * A * B *\left(t_{\mathrm{i}}-i\right)^{B-1} * e^{-A\left(t_{\mathrm{i}}-1\right)^{B}}$

$R_{\mathrm{DDS}, \text { Total }}=\sum_{i}^{\text {inftime }} R_{\mathrm{DDS}, t=i}$

The equations are adapted from Eq. 2 to account for the duration of infusion (inftime) of the DDS. During each minute of the infusion period $(t=i), R_{\mathrm{DDS}, t=i}$ starts, and the total dose released from the DDS is described with $R_{\mathrm{DDS} \text {,Total }}$.

In agreement with the observed human PK data (11), the following model settings were applied during the simulations: (i) body weight: $83 \mathrm{~kg}$ (LIPDOX) and $81 \mathrm{~kg}$ (DEBDOX); (ii) Child-Pugh class A; (iii) fraction of treated liver section $\left(F_{\mathrm{vol}, \mathrm{tr}}\right)$ : 0.093 (LIPDOX) and 0.018 (DEBDOX); (iv) embolization degree: Eqs 3 and 4 (LIPDOX) and 50\% (DEBDOX); (v) dose: $100 \mathrm{mg}$ DOX; (vi) infusion duration of DDS (inftime): $10 \mathrm{~min}$ (LIPDOX) and $13 \mathrm{~min}$ (DEBDOX).

These simulations were compared to the human PK data gathered from 13 and $12 \mathrm{HCC}$ patients receiving LIPDOX and DEBDOX, respectively (11). In this clinical study, 20 patients had Child-Pugh A liver disease, and another 5 had Child-Pugh B. All observed patient DOX PK data were dose corrected to a dose of $100 \mathrm{mg}$ DOX. The simulated and observed plasma profiles were compared visually, with PK parameters, by AAFE (Eq. 7), and by AFE (Eq. 8). Agreement between the two data sets was considered good when AAFE was $<2$, and acceptable when AAFE was $<2.5$. Note that DOXol was not included in the human PBBP model, and reasons for this are discussed in Electronic supplementary material 1 .

\section{Data Analysis and Used Software}

Curve fitting of the Weibull equation to the historical release data sets was performed with Phoenix 64 WinNonLin 6.3 (Pharsight, USA), using a user model. Different weighting schemes were tested, but only uniform weighting showed optimal curve fitting of the plateau. Therefore, the Weibull equation was fitted using the uniform weighting scheme, using the Gauss-Newton minimization function.

Agreement between data sets was evaluated with the AAFE (Eq.7) (33). The origin of the data sets being compared could be results from original and altered models, observed and simulated PK profiles, or of two simulated PK profiles. The AAFE can only be 1 or greater than 1 . An AAFE value of one indicates a perfect fit (i.e., most accurate) while a value of two indicates an average 2 -fold difference from the average observed data points.

$\mathrm{AAFE}=10 \frac{\sum\left|\log \frac{\text { new }}{\text { old }}\right|}{N}$

where new is the value given by the altered model or simulated PK profiles at a specific time point; old is the value given by the original model or historical observed $\mathrm{PK}$ profiles at that time point; and $N$ is the total number of observations.

The agreement between two data sets was also evaluated with the average fold error (AFE, Eq. 8). Here, a value of $<1$ suggests simulations resulting in lower than observed and $>1$ simulation resulting in higher than observed observations. Agreement between two data sets is shown as AAFE (or AFE), unless otherwise stated.

$A F E=10 \frac{\sum \log \frac{\text { new }}{\text { old }}}{N}$

2All simulations, i.e., pig and human in vivo PK, were performed in Berkeley Madonna (Version 8.3.18, University of California at Berkeley), using the described models (Electronic supplementary material 1) and model settings. Noncompartmental analysis was performed on the observed and simulated human data using Phoenix 64 WinNonLin 6.3 (Pharsight, USA). This analysis was executed using preset settings for the constant infusion model, except for a $1 / \mathrm{y} \times \mathrm{y}$ weighting scheme, and a Linear/Log trapezoidal computational method. PK parameters [maximum concentration $\left(C_{\max }\right)$, time to $C_{\max }\left(t_{\max }\right)$, area under the plasma concentration-time curve $\left[\mathrm{AUC}_{0-24 \mathrm{~h}}(\mathrm{VF})\right.$ or $\mathrm{AUC}_{0-6 \mathrm{~h}}(\mathrm{VH})$ ], and half-life] from the non-compartmental analysis were compared by dividing values from the simulated values with the observed ones.

\section{RESULTS}

\section{Collection and Processing of LIPDOX and DEBDOX Release Data}

Twenty-five historical in vitro and in vivo release data sets of DOX from LIPDOX were collected (LIP01-25), and 28 for DEBDOX (DEB01-28) (Electronic supplementary material 2). 
The LIP1-25 data sets described the release of DOX from different LIPDOX formulations, including suspensions in Lipiodol, (un)stabilized oil-in-water and water-in-oil emulsions, and an aqueous solution. The data sets originated from in vitro studies using different methods to characterize the release of DOX: $\mu$ Diss profiler, rotating basket method, dialysis tubes/ method, shake flask method, and T-apparatus. Two data sets characterized the release of DOX from in vivo plasma profiles by deconvolution (Electronic supplementary material 2).

The DEB1-28 data sets described release of DOX from DEBDOX formulations in which the DC bead size ranged from 100-300 to 700-900 $\mu \mathrm{m}$ and the DOX loading between 5 and $37.5 \mathrm{mg} / \mathrm{ml}$. The DEB data sets were likewise obtained from in vitro and in vivo studies that used different methods to characterize the release of DOX: $\mu$ Diss profiler (free-flowing and with sample reservoir), shake flask (with and without replacement of release medium), dialysis tubes/method, USPtype II, USP type IV, T-apparatus, and finally deconvolution from in vivo plasma profiles (Electronic supplementary material 2). The diversity of formulation compositions and in vitro release methods assured robustness in the predictions of the DOX plasma concentration-time profiles.

The estimated values for the release parameters of the 53 historical in vitro and in vivo data sets are shown in Table I and Electronic supplementary material 2. The release parameters varied greatly for both the LIP and DEB data sets. For the LIP data sets, the estimated values of $A$ ranged from $3 \times 10^{-6}$ to 0.15 , from 0.51 to 3.75 for $B$, and 0.18 to 1.00 for $F_{\text {rel,max }}$ For DEB data sets, the range was between $2 \times$ $10^{-6}-0.17$ for $A, 0.55-2.17$ for $B$, and $0.06-1.00$ for $F_{\text {rel,max }}$. Time to reach $90 \%$ release $\left(t_{90 \%}\right.$; time when $A_{\text {rel }}=$ dose $\times$ $\left.F_{\text {rel,max }} \times 0.9\right)$ ranged between $0.3-190 \mathrm{~h}$ for the LIP data sets and $0.3-2500 \mathrm{~h}$ for the DEB ones. Percentage released after $6 \mathrm{~h}\left(A_{\text {rel,6 }}\right)$ ranged from 2 to $100 \%$ for both LIP and DEB.

\section{Porcine DOX and DOXol PK Profile Simulations}

Simulations of the DOX and DOXol PK profiles after one intrahepatic dose of either LIPDOX or DEBDOX were performed twice, once with $\mathrm{NC1}$ and once with $\mathrm{NC} 2$ disposition parameters. Half of the DEBDOX and 17 of 25 LIPDOX plasma concentration-time profiles were best described with the NC2 disposition parameters (Electronic supplementary material 2). Therefore, the results shown and described in the remainder of the work are the results from simulations using the NC2 disposition parameters.

For eight LIP data sets (LIP07, 08, 09, 10, 11, 12, 14, 23) and nine DEB data sets (DEB04, 08, 12, 13, 15, 24, 25, 27, 28), there was an adequate agreement between observation and simulation $(\geq 4$ PK profiles with AAFE <2.5; Fig. 2; Electronic supplementary

Table I. Estimated Values for the Shape Factors (A and B) and Released Fraction (RF). Values were Estimated by Curve Fitting the Weibull Equation to In Vitro Data Sets of Doxorubicin Release Profiles. Formulation and Release Method Specifics of each In Vitro Data Set are Described in Electronic Supplementary Material 2. The Time (h) at which 90\% Doxorubicin is Released from the Formulation is Shown $\left(t_{90 \%}\right)$

\begin{tabular}{|c|c|c|c|c|c|c|c|c|c|}
\hline Data set & $A(\mathrm{CV} \%)$ & $B(\mathrm{CV} \%)$ & $F_{\text {rel,max }}(\mathrm{CV} \%)$ & $t_{90 \%}(\mathrm{~h})$ & Data set & $A(\mathrm{CV} \%)$ & $B(\mathrm{CV} \%)$ & $F_{\text {rel,max }}(\mathrm{CV} \%)$ & $t_{90 \%}(\mathrm{~h})$ \\
\hline LIP01 & $0.0014(27)$ & $1.2(4.2)$ & $0.47(0.89)$ & 7 & DEB03 & $0.077(7.3)$ & $0.81(2.7)$ & $0.7(0.32)$ & 1.1 \\
\hline LIP02 & $0.00042(13)$ & $1.5(1.7)$ & $0.54(0.3)$ & 6.2 & DEB04 & 0.017 (11) & $0.78(5.9)$ & $0.48(8.5)$ & 8.5 \\
\hline LIP03 & 0.00033 & $1.3(4.4)$ & 0.47 (1.6) & 15 & DEB05 & $0.032(10)$ & $0.88(3.2)$ & $0.58(0.81)$ & 2.1 \\
\hline LIP04 & $0.00023(37)$ & $1.5(4.7)$ & $0.58(1.8)$ & 8.2 & DEB06 & $0.0065(73)$ & $1.2(15)$ & $0.97(4.3)$ & 2.4 \\
\hline LIP05 & $0.00029(13)$ & $1.5(1.6)$ & $0.43(0.44)$ & 5.6 & DEB07 & $0.004(39)$ & $1.1(7.7)$ & $0.98(2.8)$ & 4.6 \\
\hline LIP06 & $0.00076(37)$ & $1.3(5.4)$ & $0.62(1.9)$ & 6.4 & DEB08 & $0.0042(53)$ & $0.87(12)$ & $1(7.7)$ & 23 \\
\hline LIP07 & $0.024(23)$ & $1.2(8)$ & $0.82(4.2)$ & 0.75 & DEB09 & 0.008 (98) & $0.84(24)$ & 0.98 (13) & 14 \\
\hline LIP08 & $0.062(4.8)$ & $0.84(3.7)$ & $0.92(3.7)$ & 1.2 & DEB10 & $0.0000025(980)$ & $2.2(77)$ & $0.95(10)$ & 9.3 \\
\hline LIP09 & $0.057(5.6)$ & $0.85(4.2)$ & $0.93(4.4)$ & 1.3 & DEB11 & $0.0003(69)$ & $0.96(9.4)$ & $1(4.4)$ & 180 \\
\hline LIP10 & $0.069(4.3)$ & $0.84(3.1)$ & $0.91(2.6)$ & 1.1 & DEB12 & $0.0067(59)$ & $0.69(16)$ & $1(11)$ & 77 \\
\hline LIP11 & 0.033 (11) & $1.5(3.3)$ & $0.95(0.6)$ & 0.3 & DEB13 & $0.00067(46)$ & $0.91(6.5)$ & 0.98 (1.6) & 130 \\
\hline LIP12 & $0.0068(9.1)$ & $1.2(2)$ & $1(0.55)$ & 2.4 & DEB14 & $0.00014(65)$ & $0.95(8.2)$ & $1(4.9)$ & 460 \\
\hline LIP13 & $0.15(7.6)$ & $0.51(8.3)$ & $0.4(6.1)$ & 3.3 & DEB15 & $0.0088(41)$ & $0.79(11)$ & $0.22(6.5)$ & 20 \\
\hline LIP14 & $0.11(31)$ & $0.64(21)$ & $1(11)$ & 2.1 & DEB16 & $0.00063(32)$ & $1.1(7.3)$ & $0.14(13)$ & 39 \\
\hline LIP15 & $0.0016(110)$ & $1.2(20)$ & $1(12)$ & 5.8 & DEB17 & $0.17(4.5)$ & $0.91(2.3)$ & $0.96(0.22)$ & 0.28 \\
\hline LIP16 & $0.0035(58)$ & $0.93(11)$ & $0.87(2.6)$ & 17 & DEB18 & $0.035(22)$ & $1.3(6.8)$ & $0.97(0.85)$ & 0.43 \\
\hline LIP17 & $0.0051(28)$ & $0.79(6.7)$ & $0.87(3.5)$ & 39 & DEB19 & $0.03(10)$ & $1.1(3.1)$ & $1(0.57)$ & 0.96 \\
\hline LIP18 & $0.00076(34)$ & $1(5.7)$ & $0.68(3.4)$ & 48 & DEB20 & $0.012(9.1)$ & $1.1(3.2)$ & $1(3.4)$ & 2.2 \\
\hline LIP19 & $0.003(11)$ & $1(2.2)$ & $1(1)$ & 13 & DEB21 & $0.0032(290)$ & $0.55(20)$ & $0.77(340)$ & 2500 \\
\hline LIP20 & $0.0081(45)$ & $0.74(12)$ & $0.79(5.3)$ & 35 & DEB22 & $0.0058(41)$ & $0.71(20)$ & $0.08(41)$ & 73 \\
\hline LIP21 & $0.0074(44)$ & $0.73(12)$ & $0.6(7.1)$ & 43 & DEB23 & $0.0046(19)$ & $0.63(4.7)$ & $0.11(31)$ & 310 \\
\hline LIP22 & $0.0032(46)$ & $0.94(8.9)$ & 0.97 (2) & 18 & DEB24 & $0.0017(27)$ & $0.97(4.4)$ & $0.27(0.94)$ & 28 \\
\hline LIP23 & $0.000003(11)$ & $3.8(0.89)$ & $1(0.14)$ & 0.62 & DEB25 & $0.013(9)$ & $0.61(6)$ & $0.27(12)$ & 79 \\
\hline LIP24 & $0.0033(72)$ & $0.98(13)$ & $0.24(3)$ & 13 & DEB26 & $0.0092(29)$ & $0.74(9.1)$ & $0.06(9)$ & 30 \\
\hline LIP25 & $0.0012(31)$ & $0.81(10)$ & $0.18(27)$ & 190 & DEB27 & $0.0099(9)$ & $0.98(2.5)$ & 0.18 (1.6) & 4.4 \\
\hline DEB01 & $0.018(2.8)$ & $1(0.71)$ & $0.78(0.15)$ & 1.9 & DEB28 & $0.0081(14)$ & $1.1(3.4)$ & $0.32(1.5)$ & 3.3 \\
\hline DEB02 & $0.06(7.4)$ & $0.82(2.6)$ & $0.64(0.4)$ & 1.5 & & & & & \\
\hline
\end{tabular}

$D E B X X$ data set where the release of doxorubicin from DC Bead was studied in vitro or in vivo, LIPXX data set where the release of doxorubicin from Lipiodol formulations was studied in vitro or in vivo 

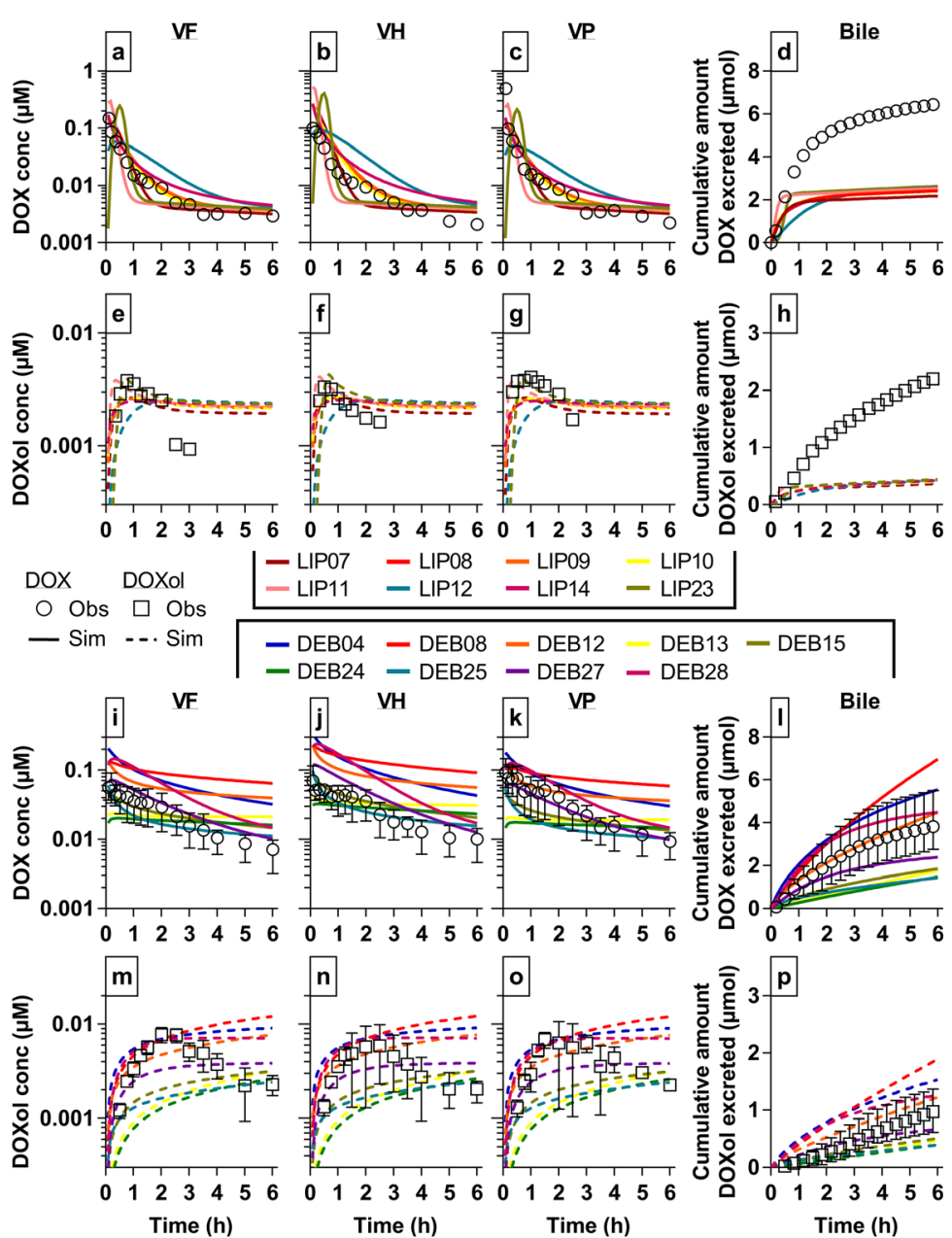

Fig. 2. Observed and simulated plasma concentration-time and biliary excretion profiles of doxorubicin (DOX) and doxorubicinol (DOXol) in healthy pigs after administration of LIPDOX (a-h) or DEBDOX (i-p) using NC2 disposition parameters. Plasma concentrations over time of DOX and DOXol in the vena femoralis (VF; $\mathbf{a}, \mathbf{e}, \mathbf{i}, \mathbf{m})$, vena hepatica $(\mathrm{VH} ; \mathbf{b}, \mathbf{f}, \mathbf{j}, \mathbf{n})$, and vena porta (VP; $, \mathbf{c}, \mathbf{g}$, $\mathbf{k}, \mathbf{o})$ are shown. Cumulative amount DOX or DOXol excreted into bile are shown in parts $\mathbf{d}, \mathbf{h}, \mathbf{l}$, and $\mathbf{p}$. Observed data ( $n=1$ for LIPDOX and $n=4$ for DEBDOX) are from (17) and simulations were performed with the pig physiologically based biopharmaceutical model

material 2). These data sets were subsequently used in the human simulations. An acceptable agreement between simulated and observed $\mathrm{PK}$ profiles (AAFE $<2.5$ for $\geq 5 \mathrm{PK}$ profiles) was obtained for seven of the eight LIP (not LIP12) and five of the nine DEB (not DEB04, 08, 28, and 29) data sets. The maximum fraction released $\left(F_{\text {rel,max }}\right)$ was estimated to be $>0.8$ for these LIP data sets (Electronic supplementary material $2)$. The percentage released after $6 \mathrm{~h}\left(A_{\text {rel, } 6 \mathrm{~h}}\right)$ was $>80 \%$ for these seven LIP data sets and time to reach $90 \%$ release $\left(t_{90 \%}\right)$ was $<$ $2.4 \mathrm{~h}$ (Electronic supplementary material 2). The five DEB data sets had an estimated $A_{\text {rel, } 6 \text { h }}$ of $10-32 \%$ DOX, while the excluded DEB data sets had either higher or lower percent release (Electronic supplementary material 2). Time to reach 90\% release $\left(t_{90 \%}\right)$ ranged from $3.3-130 \mathrm{~h}$ for these DEB data sets.

\section{Human HCC DOX PK Profile Simulations}

Simulations were performed using the PBBP model with the estimated DOX release parameters from LIP and DEB data sets that had an adequately agreement with observed porcine PK profiles. The observed human in vivo plasma concentration-time curves and simulations using these LIP (LIP07, 08, 09, 10, 11, 12, 14, 23) and DEB (DEB04, 08, 12, 13, 15, 24, 25, 27, 28) data sets are shown in Fig. 3.

Simulations using estimates from five of the eight LIP data sets (LIP07, 08, 09, 10, 14) were in good agreement with DOX plasma profiles (AAFE $<2$ in VF and VH; Fig. 3a-c; Electronic supplementary material 2). For these five LIPDOX simulations, the DOX plasma curves in VF were better described than the $\mathrm{VH}$ ones. VF plasma profiles were slightly underestimated (AFE 0.75-0.99), while VH plasma profiles were overestimated (AFE 1.2-1.8). $C_{\max }$ was 2-fold underestimated in $\mathrm{VF}$ and 1.2-fold overestimated in $\mathrm{VH}$. Data sets LIP07, 08, 09, 10, and 14 (good agreement) all had time to $90 \%$ release $\left(t_{90 \%}\right)$ between 0.8 and $2.1 \mathrm{~h}$. Simulations using LIP11 and LIP12 data sets described DOX plasma profiles with acceptable accuracy (AAFE $<2.5$ in VF and VH; Fig. 3a-c). $C_{\max }$ in VF was well described with LIP11, 

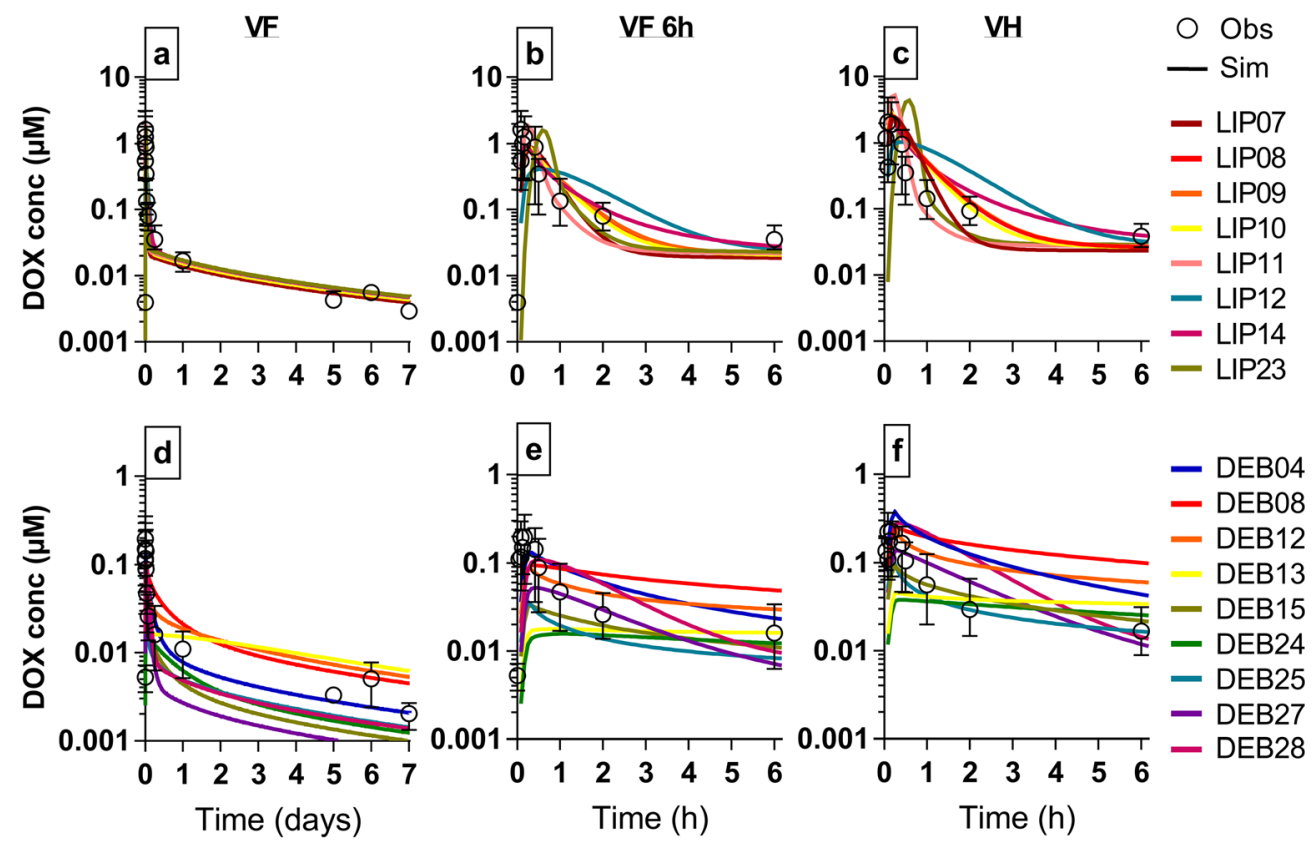

Fig. 3. Observed and simulated doxorubicin (DOX) plasma concentration-time profiles in human HCC patients after administration of LIPDOX $(n=13, \mathbf{a}-\mathbf{c})$ or $\operatorname{DEBDOX}(n=12, \mathbf{d}-\mathbf{f})$. Concentrations over time of DOX in the vena femoralis (VF; $\mathbf{a}, \mathbf{b}, \mathbf{d}, \mathbf{e})$ and vena hepatica $(\mathrm{VH} ; \mathbf{c}, \mathbf{f})$ are reported. Parts $\mathbf{b}$ and $\mathbf{e}$ show the first $6 \mathrm{~h}$ of DOX and DOXol concentrations in VF. Observed data are from (11) and simulations were performed with the human hepatocellular carcinoma physiologically based biopharmaceutical model

but 4-fold underestimated with LIP12. LIP23 underestimated the DOX plasma concentrations by 4- to 5-fold (AFE 0.4; Fig. $3 \mathrm{a}-\mathrm{c}$ ), and $t_{\max }$ was 2.2 -fold overestimated.

Simulations using estimates from DEB12 and DEB28 data sets (two out of nine tested) resulted in good agreement (AAFE $<2$ in $\mathrm{VF}$ and $\mathrm{VH}$ ) with DOX plasma profiles (Fig. 3d-f, Electronic supplementary material 2). These two in vitro data sets were calculated to have released $32 \%$ of their loaded dose after $6 \mathrm{~h}\left(A_{\text {rel,6 }} \mathrm{h}\right)$. Simulations using DEB04 and 08 gave acceptable agreement with DOX plasma curves (AAFE $<2.5$ in $\mathrm{VF}$ and $\mathrm{VH}$ ). $A_{\text {rel, } 6 \mathrm{~h}}$ was calculated to be 40 and $51 \%$ for DEB04 and DEB08, respectively. Simulations using the other five DEB data sets (DEB12, 13, $15,24,25,27,28)$ underestimated the DOX plasma concentrations. The calculated $A_{\text {rel, } 6 \mathrm{~h}}$ from these five DEB data sets was $\leq 17 \%$. All tested DEB data sets underestimated the peak plasma concentration $\left(C_{\mathrm{max}, \mathrm{Sim} / \mathrm{Obs}} 0.08-0.68\right)$. Overestimations of $\mathrm{AUC}_{0-1 \text { day }}$ were made by $\mathrm{DEB}$ data sets with an estimated $A_{\text {rel,6 }}$ h over $32 \%$, while underestimations were made by DEB data sets with estimated $A_{\text {rel, } 6 \text { h }}$ under $32 \%$.

\section{DISCUSSION}

The rate and extent of DOX release from the DDS determines the subsequent local (tumor) and systemic (plasma) concentration-time profiles. The PK profiles of DOX could be accurately described with our porcine and human HCC PBBP models using historical release data sets as input.

\section{Model Development and Performance}

The PBBP model development was described and discussed in Electronic supplementary material 1. In short, first model alterations (GI tract binding capacity, division of liver, addition of administration site to treated liver section) did adhere to the set boundaries and did not result in significant changes in DOX or DOXol PK profiles. Note that no curve fitting, i.e., parameter estimation was performed during these alterations. Thus, the porcine PBBP model described intravenous bolus doses as well as the porcine semi-PBPK model, and thus had equally good model performance. Note furthermore that two sets of DOX and DOXol disposition parameters are available: from the $\mathrm{NC} 1$ and the NC2 study. The differences in disposition are visible in the DOX biliary excretion (1 of the compared PK profiles) and DOXol plasma concentration (3 of the compared PK profiles). There was no intravenous bolus doses administered to the pigs receiving a DDS in the $\mathrm{NC1}$ study, and it was therefore unknown which disposition parameter set would best describe the disposition of DOX and DOXol in these pigs. Two simulations were thus performed for each historical release data set, one with each disposition parameter set. Selection of the best describing disposition parameter set was based on comparison of lowest AAFE for each compared compartment (DOX and DOXol in plasma (vena hepatica $(\mathrm{VH})$, vena porta (VP), vena femoralis $(\mathrm{VF})$ ) and bile). It is therefore possible that selection is skewed towards the better fitting DOXol plasma concentrations. This is for example visible in the simulations of the LIPDOX administrations, where the biliary excretion is lower with the $\mathrm{NC} 2$ than the $\mathrm{NC1}$ disposition parameters (Electronic supplementary material 2). However, DOXol plasma curves are better described with the NC2 disposition parameters. Other parameters affecting the simulations of the observed plasma and biliary excretion, such as formulation characteristics and release method properties, are described in the sections below.

Furthermore, translation from pig to human physiology, but without altering drug-specific parameters, resulted in excellent description of DOX plasma profiles. This suggests 
that the pig is an acceptable model for PK studies of DOX in humans. Our final model alterations further suggested that cirrhosis and primary liver tumors do not alter DOX plasma PK significantly. DOXol plasma profiles were not well described in the human PBBP models. This is possibly due to model misspecifications, and this part of the model was already discussed in the previously published porcine semiPBPK model paper (22). The human HCC patient PBBP model thus only described DOX PK.

\section{LIPDOX}

The simulations of porcine DOX and DOXol PK profiles described observed data best when the maximum release of DOX in the LIP release data set was over $80 \%\left(F_{\text {rel,max }}>\right.$ $0.8)$. A complete release of DOX from the DDS is also to be expected, as components of LIPDOX (water, contrast agent, Lipiodol) most likely do not bind or trap DOX. Complete release of DOX from LIPDOX in healthy pigs has been previously observed $(17,34)$, confirming and validating our simulations. In pigs and human $\mathrm{HCC}$ patients, the in vivo release rate of DOX from LIPDOX is highest immediately after the start of administration, which is followed by a subsequent decline $(11,17,35)$. Twenty minutes after start of administration, $50 \%$ of DOX has already been released to the local blood sampling site in healthy pig (vena hepatica) and in HCC patients (orifice vena hepatica and vena cava) $(11,17,35)$. This suggests rapid release of DOX from LIPDOX, independent of liver condition. In agreement with these observed data, the best descriptive human simulations from LIP data sets (LIP07-10) suggested that the time to $90 \%$ release $\left(t_{90 \%}\right)$ of the loaded DOX dose from LIPDOX takes place within 0.8 to $2.1 \mathrm{~h}$ after administration.

LIPDOX emulsions can be stable more than $24 \mathrm{~h}$ on the bench, with the use of a densifier, such as an aqueous contrast agent [Ahnfelt, personal communication] (24,36). In vivo, however, LIPDOX emulsions will mix instantaneously with the blood after locoregional administration, forming oil droplets containing water droplets (37). The emulsion separates when exposed to active blood flow; water droplets are washed away leaving only Lipiodol droplets (37). It is thus possible that the aqueous part of the emulsion behaves as an intra-arterial aqueous infusion, while the Lipiodol phase behaves as a sustained release delivery system. It has to be noted though that the amount of DOX released from Lipiodol would be low, as only up to $\sim 20 \%$ of DOX partitions to Lipiodol during $1 \mathrm{~h}$, and up to $10 \%$ during 15 min (38)[Ahnfelt, personal communication]. This is, again, formulation specific and higher for more stable emulsions. In the clinic, the emulsion is prepared extemporaneously just prior administration, by repeatedly pumping the two phases between two syringes. Time between preparation and administration is short $(<15 \mathrm{~min})$, leaving little time for DOX partitioning into the Lipiodol phase.

When the in vivo release from a DDS is fast, as it is for LIPDOX, it can be difficult to determine the best descriptive simulation of the in vivo data and thus, the best correlating in vitro release method. Without an extensive early PK profile, the simulated PK profiles based on in vitro release data can only approach the true amount released. This is partially due to limitations of in vivo studies. One such limitation is sparse blood sampling in patients, which makes it difficult to determine the true $C_{\max }$ and $t_{\max }$ values. Furthermore, the PBBP model did include the embolizing effect of Lipiodol on the microenviroment, but it did not take into account possible effects of Lipiodol on hepatic DOX disposition (39). Lipiodol might increase the intracellular concentration of DOX in pigs (17), but due to uncertainties behind the mechanism(s) of this increased concentration and its effect over time, we did not include this feature in our PBBP model. This could have led to underestimation of the local tissue concentrations and biliary excretion.

Despite the limitations in clinical data and PBBP model, the suitability of each in vitro release methods used for this type of DDS to use for simulations with the PBBP model could be assessed. In short, all LIP data sets originating from a method using a diffusion membrane (e.g., dialysis membrane) did not accurately describe the observed porcine DOX and DOXol plasma concentration-time curves and biliary excretion. A diffusion barrier can retard the diffusion of the dissolved drug substance from a formulation into the in vitro release medium - the smaller the pores in the diffusion barrier, the greater the impact on the diffusion rate of the drug substance $(16,40,41)$. When a solution is administered into the bloodstream, complete distribution of the already dissolved drug is expected to be instantaneous. The delay of release caused by a diffusion barrier will consequently be an artifact of the in vitro methodology and does not reflect in vivo conditions. Nevertheless, in vitro methods using diffusion barriers may be used for establishing a rank order of release from LIPDOX formulations. However, to establish an accurate in vivo simulation, the resulting release profiles should be corrected for the reduced release rate caused by the dialysis membrane.

\section{DEBDOX}

The most accurate descriptive simulations of the observed porcine DOX plasma concentration-time profiles were from DEB data sets with a calculated $10-32 \%$ release of loaded DOX from DEBDOX during the first $6 \mathrm{~h}$ after administration $\left(A_{\text {rel,6 }}\right.$ ). The best descriptive simulations for the human data showed around $32 \%$ release. A prolonged release from DEBDOX is in agreement with in vivo studies for DOX left in DEBDOX in situ (42-44). For instance, in pigs, measurable concentrations of DOX in DEBDOX were measurable up to 90 days, although the concentration DOX in DEBDOX decreases over time (43).

The most accurate descriptive human simulations were obtained using historical release data set DEB12. This data set described DEBDOX ${ }_{100-300 \mu \mathrm{m}}$ loaded with $5 \mathrm{mg} / \mathrm{ml}$ (23), while the observed human data used DEBDOX $_{70-500 \mu \mathrm{m}}$ loaded with $37.5 \mathrm{mg} / \mathrm{ml}$ (11). Although several ranges of bead sizes were used in the HCC patients, the beads used in DEB12 were still within the range for the observed human data set (11). As the absolute release rate is not altered by a smaller DEBDOX (100-300 $\mu \mathrm{m})$ loading dose, the difference in loaded doses between the DEB12 data set and in vivo study is of no importance (9). Interestingly, the in vitro method for DEB12 used $0.09 \% \mathrm{NaCl}$ solution as a release medium, which is lower than the physiological ion concentration $(0.9 \% \mathrm{NaCl}, 308 \mathrm{ml} / \mathrm{L})$. DOX is proposed to be released from the DC bead via an ion-exchange mechanism, i.e., when 
a positively charged counter-ion $\left(\mathrm{Na}^{+}\right)$is present (15). The lower ion concentration in the in vitro release medium of DEB12 suggests that the local in vivo environment has a decreased availability of counter-ions. This lower availability might be a consequence of blood vessel stasis caused by the administration of DEBDOX. However, as other mechanism(s) also affect the release of DOX from DEBDOX, more research on these factors is needed (19).

Interestingly, the in vitro release parameter estimates further suggested that it would take $77 \mathrm{~h}$ for $90 \%$ release from the DEB12 data set. However, a complete in vivo release is not obtained after $77 \mathrm{~h}$; DEBDOX still can contain up to $42.9 \%$ of the loaded dose as long as 80 days after administration (42). This difference in extent of release might be explained by the difference in in vitro and in vivo models. In vivo, the dispersion or clustering of individual DEBDOX beads in the blood vessel affects the release of DOX. DEBDOX clusters with more than 10 DC beads have a 10 to 20-fold higher remaining percentage than clusters with fewer beads (42). In vitro, this clustering and the effect on release have not been specifically investigated. As this could be an important factor for in vivo release, it should be included in in vitro methods describing the release of DOX from DEBDOX. With regard to physiological changes, fibrotic tissue can be formed around DEBDOX particles (a foreign body reaction) (44), which may also reduce the release rate. Our PBBP model did not take into account possible foreign body reactions. However, this PBBP model described the plasma PK of DOX up to 1 week of release from DEBDOX, and the establishment of the fibrotic tissue around a foreign particle can take up to 3 weeks (45).

Two in vitro release methods, T-apparatus and $\mu$ Diss (free flowing), do not use a diffusion membrane. As discussed in the LIPDOX section, diffusion membranes can hamper the release rate $(14,19,25,26)$. Unfortunately, data sets from these methods did not give better descriptions of the observations than those from other in vitro methods. This suggests that the diffusion barrier is less important for the release of DOX from DEBDOX than from LIPDOX, possibly because of the slower release rate. If the release rate is the rate determining step, the rate of transport over the membrane will not affect the observed appearance of drug into the release medium.

Finally, it was more difficult to describe the DOX peak plasma concentration after administration of DEBDOX to humans than to pigs. One difference between these two studies was the administration time. In the pigs, DEBDOX was administered within $2 \mathrm{~min}$, but within 4-34 min for the HCC patients. Therefore, the peak plasma concentration was at different time points for the patients. As our simulations were compared to the average DOX plasma concentration, the variability in administration time could have affected the peak plasma concentration more in humans than in pigs. Unfortunately, outside of the scope of this report, it might be interesting to simulate DOX PK profiles of individual patients in future studies. Furthermore, in the clinical study (11), DEBDOX was mixed with contrast agent and saline solution prior to administration. This might cause a small part of the loaded DOX to be released already before administration to the patient. Since this is not included in historical release data sets, the peak plasma concentration and early plasma concentrations might be described lower than observed.
In conclusion, the in vivo release of $\mathrm{DOX}$ from DEBDOX is highly dependent on the local microenvironment. Therefore, the in vitro release method used on DEBDOX must consider the bead size, DOX loading, release medium conditions such as ion concentration, and the in vivolike clustering of DEBDOX.

\section{Importance of Site of Measurement}

DOX plasma PK profiles after administration of LIPDOX or DEBDOX were well predicted when using DEB and LIP release data sets from deconvoluted local (VH) plasma curves of pigs (LIP14 and DEB28). Release profiles from deconvoluted peripheral (VF) plasma curves of pigs (LIP13 and DEB27) underestimated the human VF plasma concentration-time profiles. One possible explanation is that the $\mathrm{VH}$ sampling site is closer to the administration site in the liver, while the VF sampling site is located in the right groin. As DOX is rapidly distributed extravascularly and accumulates in tissue, the concentration in plasma will be reduced before reaching the VF sampling site. This could also explain the $\sim 2$-fold decrease in $F_{\text {rel,max }}$ for $\mathrm{VH}$ and $\mathrm{VF}$ (17). If deconvolution of plasma curves is to be used to estimate the release rate from a parenteral DDS after a single dose, we suggest sampling of the blood as close as possible to the administration site. This again shows the strength of the combination of PBBP modeling and release data sets.

\section{CONCLUSION}

Our PBBP models were able to describe DOX PK profiles of observed porcine and human (HCC) patient data. Model translation from porcine semi-PBPK to human HCC patient PBBP model was successful. This suggested that the pig is a good PK model for human HCC patients treated with LIPDOX or DEBDOX and might be useful in future formulation work.

The LIP and DEB data sets described the in vivo porcine and human acceptably. The simulations, which used diverse LIP and DEB release data sets, identified several important properties for in vitro release methods. For LIPDOX, no diffusion barriers should be used, but if they are used, the data need to be corrected for the possible decrease of release rate. For DEBDOX, the bead size and loading, ion concentration in release media, and the in vivo clustering of the beads should be taken into consideration. When evaluating the release of novel DDSs for HCC, the in vivo properties affecting drug release should be considered, before the in vitro release profile can be used to predict in vivo PK profiles.

When optimal in vitro methods for parenteral formulations are devised, the PBBP model will be an interesting tool to quickly obtain an indication of local and systemic pharmacokinetics of DOX after intra-arterial administration of a DDS. Ideally, the PBBP model will be linked to a pharmacodynamics model, to be able to describe the effect of the in vivo release and local and systemic pharmacokinetics on the disease progress. When a pharmacodynamics model is added, insight into the effect of different in vivo release profiles on the disease progression might be obtained. This could lead to the optimization or development of novel DDS 
for the treatment of HCC, where release profiles could be optimized to obtain optimal disease control.

Open Access This article is distributed under the terms of the Creative Commons Attribution 4.0 International License (http://creativecommons.org/licenses/by/4.0/), which permits unrestricted use, distribution, and reproduction in any medium, provided you give appropriate credit to the original author(s) and the source, provide a link to the Creative Commons license, and indicate if changes were made.

\section{REFERENCES}

1. Lennernas H, Lindahl A, Van Peer A, Ollier C, Flanagan T, Lionberger $\mathrm{R}$, et al. In vivo predictive dissolution (IPD) and biopharmaceutical modeling and simulation: future use of modern approaches and methodologies in a regulatory context. Mol Pharm. 2017;14(4):1307-14. https://doi.org/10.1021/ acs.molpharmaceut.6b00824.

2. Jones HM, Chen Y, Gibson C, Heimbach T, Parrott N, Peters SA, et al. Physiologically based pharmacokinetic modeling in drug discovery and development: a pharmaceutical industry perspective. Clin Pharmacol Ther. 2015;97(3):247-62. https:// doi.org/10.1002/cpt.37.

3. Sjogren E, Tammela TL, Lennernas B, Taari K, Isotalo T, Malmsten LA, et al. Pharmacokinetics of an injectable modified-release 2-hydroxyflutamide formulation in the human prostate gland using a semiphysiologically based biopharmaceutical model. Mol Pharm. 2014;11(9):3097-111. https://doi.org/ 10.1021/mp5002813.

4. Kostewicz ES, Aarons L, Bergstrand M, Bolger MB, Galetin A, Hatley $\mathrm{O}$, et al. PBPK models for the prediction of in vivo performance of oral dosage forms. Eur J Pharm Sci. 2014;57:300-21. https://doi.org/10.1016/j.ejps.2013.09.008.

5. Shen J, Burgess DJ. In vitro-in vivo correlation for complex non-oral drug products: where do we stand? J Control Release. 2015;219:644-51. https://doi.org/10.1016/j.jconrel.2015.09.052.

6. Ahmed F, Perz JF, Kwong S, Jamison PM, Friedman C, Bell BP. National trends and disparities in the incidence of hepatocellular carcinoma, 1998-2003. Prev Chronic Dis. 2008;5(3):A74.

7. Ferlay J, Soerjomataram I, Dikshit R, Eser S, Mathers C, Rebelo M, et al. Cancer incidence and mortality worldwide: sources, methods and major patterns in GLOBOCAN 2012. Int J Cancer. 2015;136(5):E359-86. https://doi.org/10.1002/ijc.29210.

8. European Association for Study of L, European Organisation for R, Treatment of C. EASL-EORTC clinical practice guidelines: management of hepatocellular carcinoma. Eur J Cancer. 2012;48(5):599-641. https://doi.org/10.1016/j.ejca.2011.12.021.

9. Dubbelboer IR, Lilienberg E, Ahnfelt E, Sjogren E, Axen N, Lennernas $\mathrm{H}$. Treatment of intermediate stage hepatocellular carcinoma: a review of intrahepatic doxorubicin drug-delivery systems. Ther Deliv. 2014;5(4):447-66. https://doi.org/10.4155/tde.14.11.

10. Gaba RC, Lewandowski RJ, Hickey R, Baerlocher MO, Cohen EI, Dariushnia SR, et al. Transcatheter therapy for hepatic malignancy: standardization of terminology and reporting criteria. J Vasc Interv Radiol. 2016;27(4):457-73. https:// doi.org/10.1016/j.jvir.2015.12.752.

11. Lilienberg E, Dubbelboer IR, Karalli A, Axelsson R, Brismar TB, Ebeling Barbier C, et al. In vivo drug delivery performance of Lipiodol-based emulsion or drug-eluting beads in patients with hepatocellular carcinoma. Mol Pharm. 2017;14(2):448-58. https://doi.org/10.1021/acs.molpharmaceut.6b00886.

12. Gonzalez W, Idee JM, Ballet S. Comment on "In vivo drug delivery performance of LIPIODOL-based emulsion or drugeluting beads in patients with hepatocellular carcinoma". Mol
Pharm. 2018;15(1):332-5. https://doi.org/10.1021/ acs.molpharmaceut.7b00138.

13. Dubbelboer IR, Lilienberg E, Karalli A, Axelsson R, Brismar TB, Ebeling Barbier C, et al. Reply to "comment on 'In vivo drug delivery performance of lipiodol-based emulsion or drugeluting beads in patients with hepatocellular carcinoma'". Mol Pharm. 2018;15(1):336-40. https://doi.org/10.1021/ acs.molpharmaceut.7b00840.

14. Lewis AL, Gonzalez MV, Lloyd AW, Hall B, Tang Y, Willis SL, et al. DC bead: in vitro characterization of a drug-delivery device for transarterial chemoembolization. J Vasc Interv Radiol. 2006;17(2 Pt 1):335-42. https://doi.org/10.1097/ 01.RVI.0000195323.46152.B3.

15. Gonzalez M, Goracci L, Cruciani G, Poggesi I. Some considerations on the predictions of pharmacokinetic alterations in subjects with liver disease. Expert Opin Drug Metab Toxicol. 2014;10(10):1397-408. https://doi.org/10.1517/ 17425255.2014 .952628$.

16. Lin SY, Wu WH, Lui WY. In vitro release, pharmacokinetic and tissue distribution studies of doxorubicin hydrochloride (Adriamycin $\mathrm{HCl}$ ) encapsulated in lipiodolized w/o emulsions and w/o/w multiple emulsions. Die Pharmazie. 1992;47(6):439-43.

17. Lilienberg E, Ebeling Barbier C, Nyman R, Hedeland M, Bondesson $\mathrm{U}$, Axen $\mathrm{N}$, et al. Investigation of hepatobiliary disposition of doxorubicin following intrahepatic delivery of different dosage forms. Mol Pharm. 2014;11(1):131-44. https:// doi.org/10.1021/mp4002574.

18. Katagiri Y, Mabuchi K, Itakura T, Naora K, Iwamoto K, Nozu Y, et al. Adriamycin-lipiodol suspension for i.a. chemotherapy of hepatocellular carcinoma. Cancer Chemother Pharmacol. 1989;23(4):238-42.

19. Ahnfelt E, Sjogren E, Hansson P, Lennernas H. In vitro release mechanisms of doxorubicin from a clinical bead drug-delivery system. J Pharm Sci. 2016;105:3387-98. https://doi.org/10.1016/ j.xphs.2016.08.011.

20. Dubbelboer IR, Lilienberg E, Hedeland M, Bondesson U, PiquetteMiller M, Sjogren E, et al. The effects of lipiodol and cyclosporin A on the hepatobiliary disposition of doxorubicin in pigs. Mol Pharm. 2014;11(4):1301-13. https://doi.org/10.1021/mp4007612.

21. Lilienberg E, Dubbelboer IR, Sjogren E, Lennernas H. Lipiodol does not affect the tissue distribution of intravenous doxorubicin infusion in pigs. J Pharm Pharmacol. 2017;69(2):135-42. https:// doi.org/10.1111/jphp.12665.

22. Dubbelboer IR, Lilienberg E, Sjogren E, Lennernas H. A modelbased approach to assessing the importance of intracellular binding sites in doxorubicin disposition. Mol Pharm. 2017;14:686-98. https://doi.org/10.1021/acs.molpharmaceut.6b00974.

23. Biondi M, Fusco S, Lewis AL, Netti PA. Investigation of the mechanisms governing doxorubicin and irinotecan release from drug-eluting beads: mathematical modeling and experimental verification. J Mater Sci Mater Med. 2013;24(10):2359-70. https://doi.org/10.1007/s10856-013-4992-4.

24. Choi JW, Cho HJ, Park JH, Baek SY, Chung JW, Kim DD, et al. Comparison of drug release and pharmacokinetics after transarterial chemoembolization using diverse lipiodol emulsions and drug-eluting beads. PLoS One. 2014;9(12):e115898. https://doi.org/10.1371/journal.pone.0115898.

25. Gonzalez MV, Tang Y, Phillips GJ, Lloyd AW, Hall B, Stratford PW, et al. Doxorubicin eluting beads-2: methods for evaluating drug elution and in-vitro:in-vivo correlation. J Mater Sci Mater Med. 2008;19(2):767-75. https://doi.org/10.1007/s10856-0060040-y.

26. Jordan O, Denys A, De Baere T, Boulens N, Doelker E. Comparative study of chemoembolization loadable beads: in vitro drug release and physical properties of DC bead and hepasphere loaded with doxorubicin and irinotecan. J Vasc Interv Radiol. 2010;21(7):1084-90. https://doi.org/10.1016/ j.jvir.2010.02.042.

27. Yamashita Y, Takahashi T, Fukushima S, Nishida M, Nakano M. Experimental study of hepatic artery administration of various formulations of lipiodol with anticancer agents. Kumamoto Med J. 1987;40(2):53-65.

28. Yi SW, Kim YH, Kwon IC, Chung JW, Park JH, Choi YW, et al. Stable lipiodolized emulsions for hepatoma targeting and 
treatment by transcatheter arterial chemoembolization. J Control Release. 1998;50(1-3):135-43.

29. Dash S, Murthy PN, Nath L, Chowdhury P. Kinetic modeling on drug release from controlled drug delivery systems. Acta Pol Pharm. 2010;67(3):217-23.

30. Kan Z, Wallace S. Sinusoidal embolization: impact of iodized oil on hepatic microcirculation. J Vasc Interv Radiol. 1994;5(6):881-6.

31. Cazejust J, Bessoud B, Colignon N, Garcia-Alba C, Planche O, Menu Y. Hepatocellular carcinoma vascularization: from the most common to the lesser known arteries. Diagnostic and Interventional Imaging. 2014;95(1):27-36. https://doi.org/ 10.1016/j.diii.2013.04.015.

32. Iwazawa J, Ohue S, Mitani T, Abe H, Hashimoto N, Hamuro M, et al. Identifying feeding arteries during TACE of hepatic tumors: comparison of C-arm CT and digital subtraction angiography. AJR Am J Roentgenol. 2009;192(4):1057-63. https://doi.org/10.2214/AJR.08.1285.

33. Graham H, Walker M, Jones O, Yates J, Galetin A, Aarons L. Comparison of in-vivo and in-silico methods used for prediction of tissue: plasma partition coefficients in rat. J Pharm Pharmacol. 2012;64(3):383-96. https://doi.org/10.1111/j.20427158.2011.01429.x.

34. Lilienberg E. Biopharmaceutical evaluation of intra-arterial drug-delivery systems for liver cancer : investigations in healthy pigs and liver cancer patients [doctoral thesis, comprehensive summary]. Uppsala: Acta Universitatis Upsaliensis; 2015.

35. Dubbelboer IR. Biopharmaceutical investigations of doxorubicin formulations used in liver cancer treatment : studies in healthy pigs and liver cancer patients, combined with pharmacokinetic and biopharmaceutical modelling [doctoral thesis, comprehensive summary]. Uppsala: Acta Universitatis Upsaliensis; 2017.

36. Nakamura H, Hashimoto T, Oi H, Sawada S. Transcatheter oily chemoembolization of hepatocellular carcinoma. Radiology. 1989:170(3 Pt 1):783-6.

37. Kan Z, Wright K, Wallace S. Ethiodized oil emulsions in hepatic microcirculation: in vivo microscopy in animal models. Acad Radiol. 1997;4(4):275-82.
38. Favoulet P, Cercueil JP, Faure P, Osmak L, Isambert N, Beltramo JL, et al. Increased cytotoxicity and stability of Lipiodol-pirarubicin emulsion compared to classical doxorubicin-Lipiodol: potential advantage for chemoembolization of unresectable hepatocellular carcinoma. Anti-Cancer Drugs. 2001;12(10):801-6.

39. Gaba RC, Schwind RM, Ballet S. Mechanism of action, pharmacokinetics, efficacy, and safety of transarterial therapies using ethiodized oil: preclinical review in liver cancer models. J Vasc Interv Radiol. 2017;29:413-24. https://doi.org/10.1016/ j.jvir.2017.09.025.

40. Ahnfelt E, Sjogren E, Axen N, Lennernas H. A miniaturized in vitro release method for investigating drug-release mechanisms. Int J Pharm. 2015;486(1-2):339-49. https://doi.org/ 10.1016/j.ijpharm.2015.03.076.

41. Lin TH, Lin SY. Encapsulation and prolonged release behavior of W/O/W type multiple emulsions. J Chin Chem Soc. 1988;35(6):463-70.

42. D'Inca H, Piot O, Diebold MD, Piardi T, Marcus C, Burde F, et al. Doxorubicin drug-eluting embolic chemoembolization of hepatocellular carcinoma: study of midterm doxorubicin delivery in resected liver specimens. J Vasc Interv Radiol. 2017;28(6):804-10. https://doi.org/10.1016/j.jvir.2017.01.018.

43. Namur J, Wassef M, Millot JM, Lewis AL, Manfait M, Laurent A. Drug-eluting beads for liver embolization: concentration of doxorubicin in tissue and in beads in a pig model. J Vasc Interv Radiol. 2010;21(2):259-67. https://doi.org/10.1016/ j.jvir.2009.10.026.

44. Namur J, Citron SJ, Sellers MT, Dupuis MH, Wassef M, Manfait $\mathrm{M}$, et al. Embolization of hepatocellular carcinoma with drugeluting beads: doxorubicin tissue concentration and distribution in patient liver explants. J Hepatol. 2011;55(6):1332-8. https:// doi.org/10.1016/j.jhep.2011.03.024.

45. Ratner BD. Reducing capsular thickness and enhancing angiogenesis around implant drug release systems. J Control Release. 2002;78(1):211-8. https://doi.org/10.1016/S0168-3659(01)00502-8. 